\title{
Renal functional MRI in mice evaluated with a dual bolus of intravascular and diffusible contrast agents
}

\author{
Michael Pedersen ${ }^{1}$, Sukru Oguz Topcu ${ }^{2}$, Steven Sourbron ${ }^{3}$, Rikke Nørregaard ${ }^{2}$
}

\author{
${ }^{1}$ MR Research Center, University of Aarhus, Skejby, Denmark; \\ ${ }^{2}$ Institute of Clinical Medicine, University of Aarhus, Skejby, Denmark; \\ ${ }^{3}$ Division of Medical Physics, Leeds University Hospital, Leeds, Great Britain. \\ Email: michael@mr.au.dk
}

Received 15 February 2011; revised 12 March 2011; accepted 14 March 2011.

\begin{abstract}
Background: The aim of this study was to evaluate the feasibility of a dual-bolus protocol, where a first bolus of an intravascular tracer is used to measure perfusion, followed by a second bolus of a freely filtered gadolinium-containing agent to measure filtration capacity. Methods: The study was conducted in mice subjected to complete unilateral ureteral obstruction (UUO), and sham operated mice were used as controls. Dynamic contrast-enhanced MRI was performed 2 days after surgery. Results and discussions: Mean signal-time curves of the renal cortex, renal medulla and abdominal aorta were used to calculate the relative renal blood flow (rRBF), relative renal blood volume ( $\mathrm{rRBV}$ ), mean transit time (MTT) and the glomerular transfer rate $K^{\text {trans }}$. We demonstrated that kidneys suffering from two days of UUO showed a decrease in cortical as well as medullary rRBF compared to kidneys from sham -operated mice. Further, we found no changes in rRBV and MTT among groups, neither in the cortex nor in the medulla. The renal functional parameter $K^{\text {trans }}$ showed a tendency (but statistically insignificant) to be reduced in the obstructed kidney compared to the sham-operated mice. Conclusions: We showed our first experiences with the consecutive use of intra- and extravascularly distributed agents in a renal-diseased mouse model, allowing analysis of both functional haemo-dyamics and filtration capacity in kidneys.
\end{abstract}

Keywords: Renal Function; Dynamic Contrast-Enhanced Magnetic Resonance Imaging; Intravascular Agents; Extravascular Agents

\section{INTRODUCTION}

Ultrasmall superparamagnetic iron oxide particles (USPIO) are small particles having a vascular remanence and are taken up by the macrophages. Consequently, USPIOs have a low clearance, facilitated mainly by the Kupffer cells, resulting in a relatively large mean half-life (several hours in humans). This pharmacokinetic behaviour can also be used to reveal the intravascular characteristics (blood perfusion) as they are usually not filtered through the glomerular membrane [1,2]. In contrary, smaller molecules like most gadolinium-containing agents are freely filtered at the glomerulus and are neither excreted nor reabsorbed, and these agents are therefore suitable for measuring the filtration capacity in the kidney [3]. The aim of this study was to evaluate the feasibility of a dual-bolus protocol, where a first bolus of an intravascular tracer is used to measure perfusion, followed by a second bolus of a freely filtered gadolinium-containing agent to measure filtration capacity. The study was conducted in a renal impaired animal model using mice subjected to complete unilateral ureteral obstruction, and sham operated mice were used as controls.

\section{MATERIALS AND METHODS}

\subsection{Animal Handling}

Studies were performed using adolescent mice (C57BL/6) $(\sim 25 \mathrm{~g})$. The animals were divided in two groups: UUO group $(n=6)$ and sham operated group $(n=6)$. Each animal was anesthetized with isoflurane (Abbott Scandinavia, Solna, Sweden), and the mice were placed on a heating pad during the operation to maintain rectal temperature at $37^{\circ} \mathrm{C}-38^{\circ} \mathrm{C}$. The left ureter was exposed via an abdominal transperitoneal incision using a microscope with $\times 25$ magnification and then completely occluded with a ligature. MRI was performed 2 days after the surgical procedure. On the day of the imaging procedure, the mouse was reanaesthetized, and a polyurethane catheter was implanted in a vein tail for administration of drugs. All procedures conformed to the na- 
tional guidelines for the care and handling of animals and the published guidelines from the National Institute of Health.

\subsection{Imaging Protocol}

MRI was performed with a Philips Intera $1.5 \mathrm{~T}$ clinical system (Philips Medical Systems, Best, The Netherlands) equipped with actively shielded magnetic field gradients with a maximal amplitude of $23 \mathrm{mT} / \mathrm{m}$. RF excitation was performed using the integrated body-coil, whereas a surface coil with a diameter of $4 \mathrm{~cm}$ was employed for reception. The animal was placed supine with the kidneys placed above the imaging coil. To avoid movements of the kidneys during free breathing and magnetic susceptibility artefacts related to the adjoining bowel, an in-house-made plastic holder was used to block the movements of the kidneys and isolate them from bowel loops while avoiding compression of the parenchyma and renal vessels. High-resolution T1-weighted images were initially generated by a 3D gradient-echo pulse sequence to allow accurate discrimination between cortical and medullary components. Sequence parameters were as follows: $\mathrm{TR} / \mathrm{TE}=1000 \mathrm{~ms} / 100 \mathrm{~ms}$, slice thickness $=2 \mathrm{~mm}$, field-of-view $=13 \times 10 \mathrm{~cm}^{2}$, and acquisition and reconstruction matrix $=256 \times 256$. Number of data averages was 16 to improve the signal-to-noise ratio. A dynamic susceptibility-weighted gradient-echo sequence was applied during an iv injection of the an USPIO (Sinarem; Guerbet, Paris, France), which was administered rapidly in less than one second, corresponding to a dose of $90 \mu \mathrm{mol} \mathrm{Fe} \mathrm{pr} \mathrm{kg.} \mathrm{A} \mathrm{dynamic} \mathrm{series} \mathrm{of} 120$ images was acquired up to $120 \mathrm{~s}$ using the parameters: repetition time $(\mathrm{TR})=50 \mathrm{~ms}$, echo time $(\mathrm{TE})=20 \mathrm{~ms}$ and flip-angle $=70^{\circ}$, allowing images to be acquired with an interval of $0.6 \mathrm{~s}$. Acquired slice thickness was 2 $\mathrm{mm}$ and spatial resolution was $64 \times 128$, but was reconstructed to a $256 \times 256$ matrix to allow overlaying on the anatomical weighted image. Thirty minutes after injection of Sinerem, an iv bolus of $0.2 \mathrm{mmol} / \mathrm{kg}$ of a freely diffusible gadolinium-containing agent (Magnevist; Bayer Schering, Berlin, Germany) was administered in less than one second. A dynamic T1-weighted gradient-echo sequence was applied using the same orientation, spatial resolution, number of slices, slice thickness, and voxel size as before, and with the following parameters: imaging time was set to $180 \mathrm{~s}$ and number of averages were two to improve signal-to-noise ratio, $\mathrm{TR}=12 \mathrm{~ms}, \mathrm{TE}=$ $4 \mathrm{~ms}$, and flip-angle $=9^{\circ}$. All images were acquired in the axial plane without respiratory compensation.

\subsection{Image Analysis}

Image analysis was performed using the Mistar software package (Apollo Imaging Technology, Melbourne, Australia). Region-of-interests (ROI) were drawn in the ab- dominal aorta, renal (left) cortex and (left) medulla on the high-resolution images, and ROIs were duplicated to both the dynamic susceptibility-weighted and the dynamic contrast-enhanced images. Mean signal-time curves of the three ROIs were computed. Dynamic susceptibility-weighted (Sinerem) data were analysed using the standard model-free approach for intravascular contrast agents [4], generating the parameters relative renal blood flow (rRBF, in $\mathrm{ml} / 100 \mathrm{ml} / \mathrm{min}$ ), relative renal blood volume (rRBV, in $\mathrm{ml} / 100 \mathrm{ml}$ ), and mean transit time (MTT, in s). Dynamic contrast-enhanced (Magnevist) data were analysed assuming a linear relation between signal change and tracer concentration, and using the standard Tofts' kinetic model [5], producing the transfer constant $\left(\mathrm{K}^{\text {trans }}\right.$, in $\left.1 / \mathrm{s}\right)$ that can be a surrogate marker for the permeability across the glomerular membrane (filtration). Statistical significance for the difference between the two groups (obstructed and non-obstructed) was evaluated with an unpaired Student's t-test with equal variance, and a unpaired t-test was applied to evaluate statistical difference between contralateral (untouched) kidneys in the UUO group and sham-operated kidneys. Summarised data of rRBF, rRBV, MTT, and $\mathrm{K}^{\text {trans }}$ are presented as mean \pm standard error of mean. The statistical acceptance criterion was $p<0.05$.

\section{RESULTS}

The dual bolus measurement was performed successfully in all twelve mice. Examples of the time-concentration curves following injection of Sinerem and Gd-DTPA, respectively, are shown in Figure 1. Cortical rRBF in the obstructed kidney was decreased compared to sham cortical rRBF $(227 \pm 36$ vs $317 \pm 41 \mathrm{ml} / \mathrm{min} / 100 \mathrm{ml}$; $\mathrm{p}=$ 0.02 ), and similarly, medullary rRBF in the obstructed kidney was decreased compared to sham medullary $\operatorname{rRBF}(60 \pm 14 \mathrm{vs} 100 \pm 18 \mathrm{ml} / \mathrm{min} / 100 \mathrm{ml} ; p=0.007)$. Cortical rRBV in the obstructed kidney was not significantly changed compared to sham cortical rRBV (34 \pm 9 vs $37 \pm 7 \mathrm{ml} / 100 \mathrm{ml} ; p>0.05)$, and obstructed medullary rRBV was not significantly changed compared to sham medullary $\mathrm{rRBV}(15 \pm 2 \mathrm{vs} 20 \pm 3 \mathrm{ml} / 100$ $\mathrm{ml} ; p>0.05)$. Obstructed cortical MTT was unchanged compared to the sham group ( $9 \pm 1$ vs $7 \pm 1 \mathrm{~s} ; p>0.05)$, and similar findings were found in medulla ( $15 \pm 2$ vs 12 $\pm 1 \mathrm{~s} ; p>0.05) . \mathrm{K}^{\text {trans }}$ demonstrated no statistical changes $48 \mathrm{~h}$ after obstruction compared to sham rats (9 \pm 2 vs $\left.10 \pm 1 \mathrm{~s}^{-1} ; p>0.05\right)$. Data are presented in Figure 2. In neither case did parametric values differ among contra- lateral (untouched) kidneys in the UUO group and sham-operated kidneys $(p>0.05)$.

\section{DISCUSSION}

This experimental MRI study in mice demonstrated the feasibility of a dual perfusion train, with a first injection 


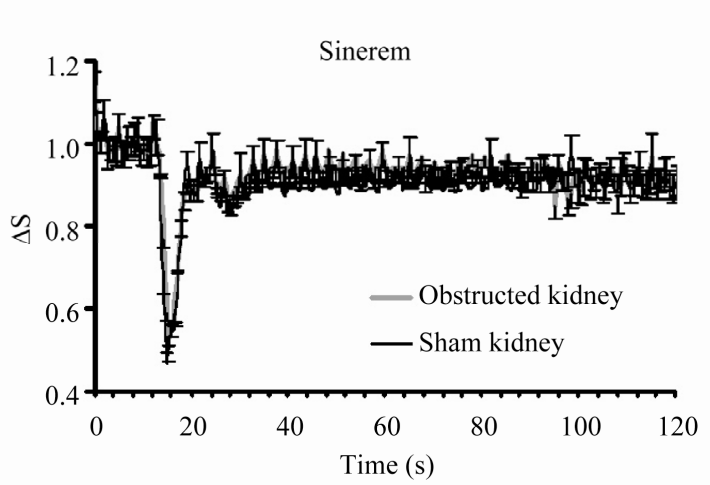

(a)

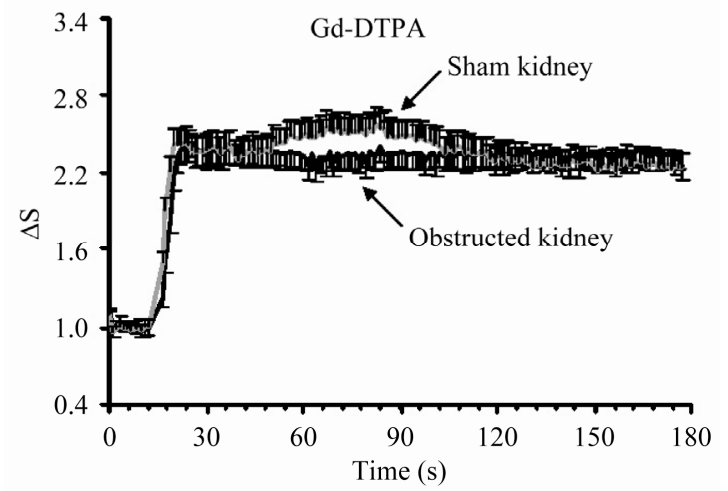

(b)

Figure 1. The relative signal intensity curves show the first of Sinerem (a) and Gd-DTPA (b) followed by continous contrast material accumulation in the mouse kidney.
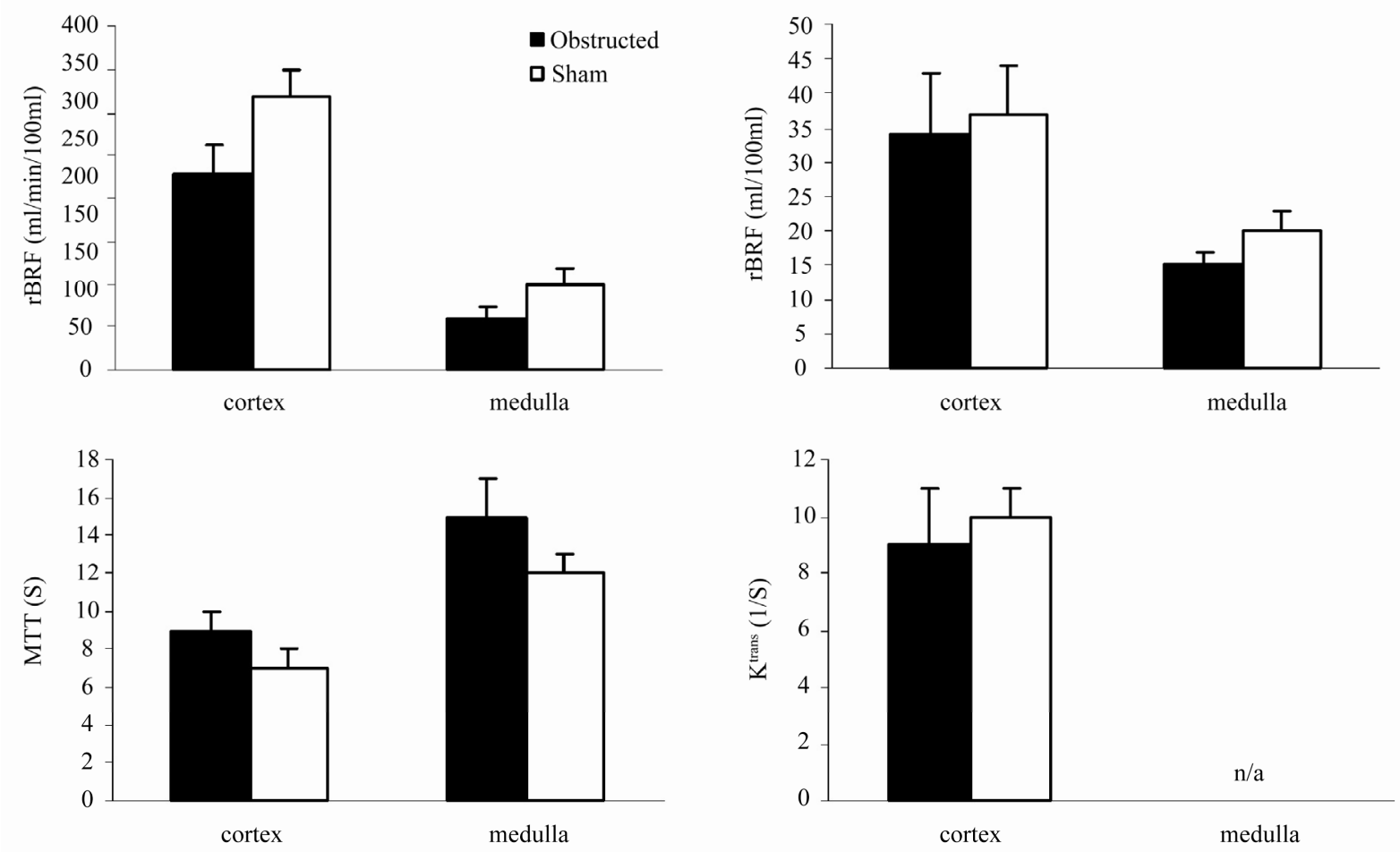

Figure 2. Parameters of cortical and medullary function measured in obstructed and sham mice: Relative renal blood flow (rRBF), relative renal blood volume (rRBV), mean transit time (MTT) and the glomerular uptake transfer rate $\mathrm{K}^{\text {trans }}$.

of an intravascularly confined agent, and a second injection of an extravasating agent. Bolus-tracking data were acquired with a dynamic susceptibility-weighted MRI and a dynamic contrast-enhanced MRI sequence, respectively. We demonstrated that kidneys suffering from two days of UUO showed a decrease in cortical as well as medullary renal blood flow (rRBF) compared to kidneys from sham-operated mice. Further, we found no changes in relative renal blood volume (rRBV) and MTT among groups, neither in the cortex nor in the medulla. The re- nal functional parameter $\mathrm{K}^{\text {trans }}$ showed a tendency (but statistically insignificant) to be reduced in the obstructed kidney compared to the sham-operated mice.

It is well-known that complete obstruction of the urinary tract has marked effects on RBF. In experimental animals, complete unilateral ureteral obstruction (CUUO) for $24 \mathrm{~h}$ causes a decrease in RBF in the obstructed kidney [6-8]. Previous studies have demonstrated that, at 24 $h$ into the obstruction, large areas of the cortical vascular bed are either underperfused or not perfused at all $[8,9]$. 
In addition, it has been observed in rats that RBF was reduced to $30 \%$ of controls 6 days after CUUO [10]. Solez et al. also demonstrated a marked reduction in inner medullary plasma flow (IMPF) in rats subjected to CUUO for $18 \mathrm{~h}$, whereas in the contralateral nonobstructed kidney IMPF was not significantly different from that in the control rats [11]. Our results are in agreement with those observations. The calculated values of rRBF and rRBV are also in the order of magnitude of those recently reported in a pcy (polycystic kidneys and fibrosis) mouse model using a clinical 3 T MRI system [12]. On the other hand, it is also known that GFR is decreased in response to CUUO. After $24 \mathrm{~h}$ of CUUO the continued fall in GFR of the obstructed kidney is associated with a compensatory GFR increase in the contralateral nonobstructed kidney [13]. Our study could not confirm these findings. A possible explanation for the lack of significant reduction in $\mathrm{K}^{\text {trans }}$ (a surrogate marker of GFR) may be an increase in glomerular filtration capacity at $48 \mathrm{~h}$ compared to the state at $24 \mathrm{~h}$. Another explanation may be the use of mice in our study instead of rats. A methodological explanation lies in the known limitations of the Tofts' model [5]. The parameter $\mathrm{K}^{\text {trans }}$ is often tacitly interpreted as measuring permeability or filtration, but it is in fact a mixture of perfusion and filtration. In certain regimes it may be interpreted as perfusion (flow-limited), in others as filtration (permeability-limited), but in most situations it will represent a combination of both effects. This may well explain the lack of specificity in our values for $\mathrm{K}^{\text {trans }}$, for instance if the obstruction induces a transition from a permeabilitylimited to a flow-limited regime, or vice versa. Moreover, it has been shown in rabbits, humans and polycystic mice $[3,12,14]$ that the Tofts' model does not provide an accurate description of the kinetics of an MR tracer with small molecular weight, measured at high temporal resolution. For such data the model must be refined by separating intra- and extravascular compartments [14]. This not only provides a more accurate description of the data, but also a separate measurement of perfusion and filtration, thereby eliminating any possible ambiguities in the interpretation of $\mathrm{K}^{\text {trans }}$. The renophysiologial and renofunctional changes following ureteral obstruction go in parallel with volumetric changes (increased pelvis and reduced cortex) and changes in mean cell density and accumulation of myofibroblasts and collagen deposition [15].

A second potential problem in the measurement of GFR with T1-weighted bolus-tracking is the precise relationship between signal change and tracer concentration. In this study, we assume a linear relationship between contrast agent concentration and measured signal change. A related issue is that of limited water exchange between intra- and extravascular space [16]. In the first pass of a small molecular agent, strong concentration differences exist between intra- and extravascular spaces. This results in strong differences in T1 at peak concentration between blood and tissue. The tissue then leaves the fast-exchange regime, and a two-compartment model for water kinetics must be used. Problems in the signal analysis may also arise in the susceptibility-weighted acquisition of the first bolus with Sinerem. A first problem is that the transverse relaxation rate $1 / \mathrm{T} 2 *$ in blood is not linear with the iron concentration [17]. Moreover, the results from the study by Bjørnerud et al indicated that the presence of $\mathrm{T} 1$ effects can lead to a significant underestimation of perfusion even in the absence of extravascular leakage, as reflected in the area and peak height of the first-pass curve following bolus injection of iron oxide nanoparticles [17]. The renal concentration time curves were deconvolved with the arterial input function (AIF) in order to compensate for the dispersion of the contrast bolus between the site of injection and the tissue of interest, as well as the finite duration of the bolus injection. The error estimated on deconvolved perfusion data with $\mathrm{T} 1$ shortening has previously been investigated by Aumann and coworkers [1], who demonstrated that rRBV and rRBF were overestimated when T1 shortening was not taken into account. Importantly, it was notwithstanding stated that the error resulting from this effect was negligible in the measurement of kidney perfusion when using certain practical considerations (dependent on sequence parameters and dose of the intravascular agent).

In conclusion, we showed our first experiences with the consecutive use of intra- and extravascularly distributed agents in a renal-diseased mouse model, allowing analysis of both functional haemodyamics and filtration capacity in kidneys. The method was employed in mice having a ureteral obstruction $48 \mathrm{~h}$ before MRI. These promising findings encourage future use of this dualbolus approach in experimental settings, where noninvasive determinations of renal perfusion and filtration are requested.

\section{ACKNOWLEDGEMENTS}

We thank Guerbet for kindly providing vials of Sinerem.

\section{REFERENCES}

[1] Aumann, S., Schoenberg S.O., Just, A., Briley-Saebo, K., Bjørnerud, A., Bock, M. and Brix, G. (2003) Quantification of renal perfusion using an intravascular contrast agent (part 1): Results in a canine model. Magnetic Resonance in Medicine, 49, 276-287.

[2] Schoenberg, S.O., Aumann S., Just, A., Bock, M., Knopp, M.V., Johansson, L.O. and Ahlstrom, H. (2003) Quanti- 
fication of renal perfusion abnormalities using an intravascular contrast agent (part 2): Results in animals and humans with renal artery stenosis. Magnetic Resonance in Medicine, 49, 288-298.

doi: $10.1002 / \mathrm{mrm} .10383$

[3] Annet, L., Hermoye, L., Peeters, F., Jamar, F., Dehoux, J.P. and Van Beers, B.E. (2004) Glomerular filtration rate: Assessment with dynamic contrast-enhanced MRI and a cortical-compartment model in the rabbit kidney. Journal of Magnetic Resonance Imaging, 20, 843-849. doi:10.1002/jmri.20173

[4] Østergaard, L., Weisskoff, R.M., Chesler, D.A., Gyldensted, C. and Rosen, B.R. (1996) High resolution measurement of cerebral blood flow using intravascular tracer bolus passages. Part I: Mathematical approach and statistical analysis. Magnetic Resonance in Medicine, 36, 715725. doi:10.1002/mrm.1910360510

[5] Tofts, P.S., Brix, G., Buckley, D.L., Evelhoch, J.L., Henderson, E., Knopp, M.V., Larsson, H.B., Lee, T.Y., Mayr, N.A., Parker, G.J., Port, R.E., Taylor, J. and Weisskoff, R.M. (1999) Estimating kinetic parameters from dynamic contrast-enhanced T(1)-weighted MRI of a diffusable tracer: Standardized quantities and symbols. Journal of Magnetic Resonance Imaging, 10, 223-232.

[6] Idbohrn, H. and Muren, R.A. (1956) Blood flow in experimental hydronephrosis. Acta Physiologica Scandinavica, 38, 200-206. doi:10.1111/j.1748-1716.1957.tb01384.x

[7] Wen, J.G., Frøkiær, J., Jørgensen, T.M. and Djurhuus, J.C. (1999) Obstructive nephropathy: An update of the experimental research. Urologica Research, 27, 29-39. doi: $10.1007 / \mathrm{s} 002400050086$

[8] Yarger, W.E. and Griffith, L.D. (1974) Intrarenal hemodynamics following chronic unilateral ureteral obstruction in the dog. American Journal of Physiology, 227, 816-826.

[9] Harris, R.H. and Gill, J.M. (1981) Changes in glomerular filtration rate during complete ureteral obstruction in rats. Kidney International, 19, 603-608.

doi:10.1038/ki.1981.58
[10] Clausen, G. and Hope, A. (1977) Intrarenal distribution of blood flow and glomerular filtration during chronic unilateral ureteral obstruction. Acta Physiologica Scandinavica, 100, 22-32.

[11] Solez, K., Ponchak, S., Buono, R.A., Vernon, N., Finer, P.M., Miller, M. and Heptinstall, R.H. (1976) Inner medullary plasma flow in the kidney with ureteral obstruction. American Journal of Physiology, 231, 1315-1321.

[12] Sadick, M., Schock, D., Kraenzlin, B., Gretz, N., Schoenberg, S.O. and Michaely, H.J. (2009) Morphologic and dynamic renal imaging with assessment of glomerular filtration rate in a pcy-mouse model using a clinical 3.0 Tesla scanner. Investigative Radiology, 44, 469-475. doi:10.1097/RLI.0b013e3181a8afa1

[13] Harris, R.H. and Yarger, W.E. (1974) Renal function after release of unilateral ureteral obstruction in rats. American Journal of Physiology, 227, 806-815.

[14] Sourbron, S.P., Michaely, H.J., Reiser, M.F. and Schoenberg, S.O. (2008) MRI-measurement of perfusion and glomerular filtration in the human kidney with a separable compartment model. Investigative Radiology, 43, 4048. doi:10.1097/RLI.0b013e31815597c5

[15] Togao, O., Doi, S., Kuro, M., Masaki, T., Yorioka, N. and Takahashi, M. (2010) Assessment of renal fibrosis with diffusion-weighted MR imaging: Study with murine model of unilateral ureteral obstruction. Radiology, 255, 772780. doi:10.1148/radiol.10091735

[16] Buckley, D.L., Kershaw, L.E. and Stanisz, G.J. (2008) Cellular-interstitial water exchange and its effect on the determination of contrast agent concentration in vivo: dynamic contrast-enhanced MRI of human internal obturator muscle. Magnetic Resonance in Medicine, 60, 1011-1019. doi:10.1002/mrm.21748

[17] Bjørnerud, A., Johansson, L.O., Briley-Saebo, K. and Ahlstrom, H.K. (2002) Assessment of T1 and T2* effects in vivo and ex vivo using iron oxide nanoparticles in steady state-dependence on blood volume and water exchange. Magnetic Resonance in Medicine, 47, 461471. 